\title{
OPEN A novel prognostic signature of immune-related IncRNA pairs in lung adenocarcinoma
}

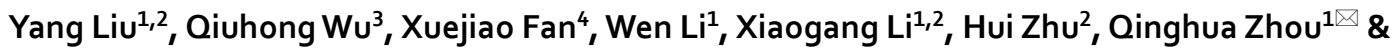 \\ Jinming $\mathrm{Yu}^{2 \bowtie}$
}

Lung adenocarcinoma (LUAD) is the most common subtype of lung cancer, but the prognosis of LUAD patients remains unsatisfactory. Here, we retrieved the RNA-seq data of LUAD cohort from The Cancer Genome Atlas (TCGA) database and then identified differentially expressed immune-related IncRNAs (DEirlncRNAs) between LUAD and normal controls. Based on a new method of cyclically single pairing along with a 0 -or- 1 matrix, we constructed a novel prognostic signature of 8 DEirlncRNA pairs in LUAD with no dependence upon specific expression levels of IncRNAs. This prognostic model exhibited significant power in distinguishing good or poor prognosis of LUAD patients and the values of the area under the curve (AUC) were all over 0.70 in 1, 3, 5 years receiver operating characteristic (ROC) curves. Moreover, the risk score of the model could serve as an independent prognostic factor for patients with LUAD. In addition, the risk model was significantly associated with clinicopathological characteristics, tumor-infiltrating immune cells, immune-related molecules and sensitivity of anti-tumor drugs. This novel signature of DEirlncRNA pairs in LUAD, which did not require specific expression levels of IncRNAs, might be used to guide the administration of patients with LUAD in clinical practice.

Lung cancer remains the leading cause of cancer-related deaths worldwide. Non-small-cell lung cancer (NSCLC) accounts for $80 \%$ of lung cancer cases, of which lung adenocarcinoma (LUAD) is the most common subtype ${ }^{1,2}$. LUAD patients are often diagnosed at advanced disease stage. Despite advances have been achieved in the treatment of advanced LUAD, such as molecularly targeted therapy and immunotherapy, the overall survival (OS) of advanced LUAD patients is still unsatisfactory, with 5-year OS rate less than $20 \%^{3-5}$. Therefore, it is urgent to develop efficient biomarkers to accurately predict the prognosis of LUAD patients.

In recent years, tumor microenvironment (TME) has been proved to be involved in the occurrence and development of malignant tumors ${ }^{6,7}$. And cancer cells possess various immune resistance mechanisms to escape the surveillance and elimination of anti-tumor immunity, namely immunoediting, such as downregulation of costimulatory molecules and major histocompatibility complex (MHC), upregulation of immunosuppressive ligands, and lack of appropriate inflammatory cytokines to attract anti-tumor immune cells ${ }^{8}$. In addition, there are also some inhibitory immune cells in the TME, such as regulatory T cells (Tregs) and myelogenic suppressor cells (MDSCs), which are also the reasons for cancer cells to escape immune surveillance ${ }^{9,10}$. Therefore, evaluating the proportion of tumor-infiltrating immune cells in the TME can not only improve our understanding of the pathogenesis of cancers, but also help us to develop novel biomarkers to predict the effectiveness of immunotherapy.

Long non-coding RNAs (lncRNAs), longer than 200 nucleotides in length, are a subtype of non-coding RNAs (ncRNAs) that can regulate gene expression at both transcriptional and post-transcriptional levels. Accumulating evidences have suggested that lncRNAs can participate in the occurrence and development of cancers ${ }^{11,12}$. In addition, lncRNAs can regulate the TME and play an essential role in tumor immunity ${ }^{13,14}$. For instance, $\mathrm{Li}$ et al. systematically analyzed immune-related lncRNAs (irlncRNAs) in 33 cancer types and found that several lncRNAs were significantly associated with immune cell infiltration ${ }^{15}$. Furthermore, recent studies have indicated that signatures of irlncRNAs could serve as prognostic biomarkers of the survival of cancers, such as breast cancer,

\footnotetext{
${ }^{1}$ Lung Cancer Center, West China Hospital, Sichuan University, Chengdu 610041, Sichuan, China. ${ }^{2}$ Department of Radiation Oncology, Shandong Cancer Hospital and Institute, Shandong First Medical University and Shandong Academy of Medical Sciences, Jinan 250117, Shandong, China. ${ }^{3}$ Department of Rheumatology and Immunology, West China Hospital, Sichuan University, Chengdu 610041, Sichuan, China. ${ }^{4}$ Clinical Research Management Department, West China Hospital, Sichuan University, Chengdu 610041, Sichuan, China. ${ }^{\square}$ email: prof_qh_ zhou@126.com; sdyujinming@163.com
} 
colon cancer, pancreatic cancer as well as $\mathrm{LUAD}^{16-20}$. By using the gene expression data and clinical data in The Cancer Genome Atlas (TCGA), Miao et al. constructed a signature of immune-related six-lncRNAs to predict the OS of patients with LUAD and this signature could act as an independent prognostic factor ${ }^{18}$. Similarly, Li et al. identified a seven irlncRNA signature for predicting OS of LUAD patients and this prognostic model could well distinguish good or poor survival of patients with LUAD ${ }^{20}$. However, the majority of these lncRNA signatures were based on the quantitative expression levels of lncRNAs. Since different experimental platforms and batches may lead to the heterogeneity of gene expression data, it is inappropriate to apply one gene signature from specific platform to another gene expression data without normalization and correction, limiting the clinical application values of these lncRNA signatures.

In this study, we downloaded the RNA-seq data and corresponding clinical information from TCGA database and constructed a novel signature of irlncRNA pairs in LUAD. Then, the predictive value of this prognostic prediction model was estimated. Considering that it only needed the relative expression levels between the two in each pair, this irlncRNA pair signature was not affected by the heterogeneity of different experimental platforms, making it more valuable for clinical application. Meanwhile, we also evaluated the correlation between this risk model and tumor-infiltrating immune cells or immune-related molecules, as well as the relationship between the model and chemotherapeutic efficacy of LUAD.

\section{Methods and materials \\ Data download and processing. The transcriptome profiling (RNA-seq) data and corresponding clinical information of LUAD samples were downloaded from TCGA database (https://portal.gdc.cancer.gov/). Patients without corresponding RNA-seq data or clinical information or with a survival time $<30$ days were excluded. GTF files were retrieved from Ensembl dataset (http://asia.ensembl.org), which were used to transform the Ensembl ID of genes into homologous gene symbols and to distinguish mRNAs and lncRNAs. And the gene list of immune-related genes (ir-genes) was obtained from the ImmPort database (http://www.immport.org).}

irlncRNAs identification and differential expression analysis. Pearson correlation analysis was used to investigate the correlation between ir-genes and lncRNAs, and the absolute value of correlation coefficient $>0.5$ and $\mathrm{P}<0.001$ were used as the criteria to identify irlncRNAs. Then, differentially expressed irlncRNAs (DEirlncRNAs) between LUAD and normal controls were identified by "limma" package of R software (v.4.0.3 $)^{21}$. The Benjamini-Hochberg (BH) false discovery rate (FDR) method was conducted to adjust $\mathrm{P}$ values ${ }^{22}$. Those irlncRNAs that meet the screening criteria $(\mid \log$ fold change $(\mathrm{FC}) \mid>2$, and adjusted $\mathrm{P}$ value $<0.05)$ were identified as DEirlncRNAs. Then, the "ggplot2" and "pheatmap" packages of R software were used to depict the volcano plot and heatmap of DEirlncRNAs.

Establishment of DEirlncRNA pairs. We cyclically single paired the DEirlncRNAs and constructed a 0-or-1 matrix. For one DEirlncRNA pair (lncRNA A|lncRNA B), if the expression level of lncRNA A was higher than lncRNA B, the value of this pair was defined as 1 . On the contrary, the value of lncRNA A|lncRNA B was defined as 0 if the expression level of lncRNA A was lower than lncRNA B. Then, those DEirlncRNA pairs with 0 -or- 1 less than $20 \%$ or more than $80 \%$ of total pairs were excluded, since only pairs with a certain rank were closely associated with the prognosis of patients.

Identification of survival-related DEirlncRNA pairs and construction of prognostic prediction model. We conducted univariate Cox regression analysis to identify survival-related DEirlncRNAs $(\mathrm{P}<0.05)$ by using "survival" and "survminer" R packages. Then, by using "glmnet" R package, we performed the least absolute shrinkage and selection operator (LASSO) regression analysis to select the most significant DEirlncRNA pairs out of all OS-related DEirlncRNA pairs. This process was performed to avoid model overfitting, and several optimal DEirlncRNA pairs with non-zero coefficients were used as candidates to construct the prognosis predictive model by multivariate Cox regression analysis ${ }^{23}$. In the risk model, the risk score of each LUAD patients was calculated based on the value of lncRNA A|lncRNA B ( 0 or 1$)$ and the corresponding multivariate Cox regression coefficient. The formula was as follows: Riskscore $=\sum_{i}^{n}(\operatorname{lncRNAA} \mid \operatorname{lncRNAB}) \mathrm{i} \beta \mathrm{i}$, where $\beta$ represented the regression coefficient.

Evaluation of prognostic prediction model. The 1-, 3-, and 5-year receiver operating characteristic (ROC) curve analyses were conducted and the values of the area under the curve (AUC) were calculated by using "survival ROC" R package to estimate the specificity and sensitivity of the model. The point of maximum Youden Index in the 1-year ROC curve were defined as the cut-off point, and its corresponding risk score was used to divide patients with LUAD into high-risk and low-risk subgroups ${ }^{24}$. The formula was as follows: YoudenIndex $=$ Sensitivity + Specificity -1 . The Kaplan-Meier $(\mathrm{K}-\mathrm{M})$ analysis and log-rank test were conducted to compare the survival difference between high-risk and low-risk patients. Then, LUAD samples were reordered based on the risk score, and the risk score curve and the survival status distribution were plotted. Furthermore, the independent prognostic roles of risk score and clinicopathological characteristics, such as age, gender, and TNM stage, were assessed by univariate and multivariate Cox regression analysis. In addition, the relationship between risk model and clinicopathological characteristics was assessed by chi-square test, which was visualized by heatmap. And Wilcoxon rank-sum test was used to compare the difference of risk scores among patients with different TNM stages. 


\begin{tabular}{|c|c|c|}
\hline Characteristics & No. of patients & $\%$ \\
\hline \multicolumn{3}{|c|}{ Age at diagnosis (years) } \\
\hline$\leq 65$ & 231 & 47.14 \\
\hline$>65$ & 249 & 50.82 \\
\hline Unknown & 10 & 2.04 \\
\hline \multicolumn{3}{|l|}{ Sex } \\
\hline Male & 228 & 46.53 \\
\hline Female & 262 & 53.47 \\
\hline \multicolumn{3}{|l|}{ Stage } \\
\hline I & 263 & 53.67 \\
\hline II & 115 & 23.47 \\
\hline III & 79 & 16.12 \\
\hline IV & 25 & 5.10 \\
\hline Unknown & 8 & 1.63 \\
\hline \multicolumn{3}{|l|}{ T category } \\
\hline $\mathrm{T} 1$ & 163 & 33.27 \\
\hline $\mathrm{T} 2$ & 263 & 53.67 \\
\hline T3 & 43 & 8.78 \\
\hline $\mathrm{T} 4$ & 18 & 3.67 \\
\hline $\mathrm{Tx}$ & 3 & 0.61 \\
\hline \multicolumn{3}{|l|}{$\mathrm{N}$ category } \\
\hline No & 317 & 64.69 \\
\hline N1 & 92 & 18.78 \\
\hline N2 & 68 & 13.88 \\
\hline N3 & 2 & 0.41 \\
\hline $\mathrm{Nx}$ & 11 & 2.24 \\
\hline \multicolumn{3}{|l|}{ M category } \\
\hline M0 & 324 & 66.12 \\
\hline M1 & 24 & 4.90 \\
\hline $\mathrm{Mx}$ & 142 & 28.98 \\
\hline
\end{tabular}

Table 1. Characteristics of patients with LUAD from TCGA database. LUAD, lung adenocarcinoma; TCGA, The Cancer Genome Atlas.

Comprehensive analysis of tumor-infiltrating immune cells and immune-related molecules. The status of immune cell infiltration of LUAD samples was estimated using the online platform of Tumor Immune Estimation Resource 2.0 (TIMER2.0, http://timer.cistrome.org/), which integrated seven quantification methods of immune infiltration estimations including TIMER, xCell, quanTIseq, MCP-counter, EPIC, CIBERSORT-ABS, and CIBERSORT ${ }^{25-27}$. Then, we performed Spearman correlation analysis to explore the relationship between risk score and tumor-infiltrating immune cells, and $\mathrm{P}<0.05$ was considered statistically significant. Furthermore, the correlation between risk model and the expression levels of genes associated with immune checkpoint inhibitors (ICIs), including cytotoxic T-lymphocyte associated protein 4 (CTLA4), programmed cell death 1 (PDCD1), lymphocyte activating 3 (LAG3), and hepatitis A virus cellular receptor 2 (HAVCR2), was evaluated by the Wilcoxon rank-sum test and visualized by "ggpubr" package in R software.

Sensitivity evaluation of anti-tumor drugs. We obtained the Immunophenoscores (IPS) of patients with LUAD from the Cancer Immunome Database (TCIA, https://tcia.at/home). And the relationship between IPS and the risk signature was investigated by the Wilcoxon rank-sum test. Furthermore, the half inhibitory concentration (IC50) of common anti-tumor drugs was analyzed by "pRRophetic" package in R software. To explore the clinical value of this risk model in the treatment of LUAD, the Wilcoxon rank-sum test was conducted to estimate the difference of IC50 of anti-tumor drugs, including paclitaxel, docetaxel, gemcitabine, vinorelbine, etoposide, cisplatin, gefitinib, and erlotinib, between the high-risk and low-risk subgroups.

\section{Results}

Identification of DEirlncRNAs. Totally, this study included 490 LUAD samples and 59 normal controls from TCGA database, and the characteristics of 490 patients with LUAD (228 men and 262 women) were shown in Table 1. The majority of patients with LUAD in TCGA were in early stages, including stage I in 263 cases (53.67\%), stage II in 115 cases (23.47\%), stage III in 79 cases (16.12\%) and stage IV in 25 cases (5.10\%). According to the co-expression analysis between lncRNAs and ir-genes, we identified 1035 irlncRNAs (Supplementary Table S1). Among them, 91 DEirlncRNAs were identified between LUAD and normal controls, of which 73 DEirlncRNAs were upregulated and 18 DEirlncRNAs were downregulated (Fig. 1A and 1B and Supplementary 
A

B
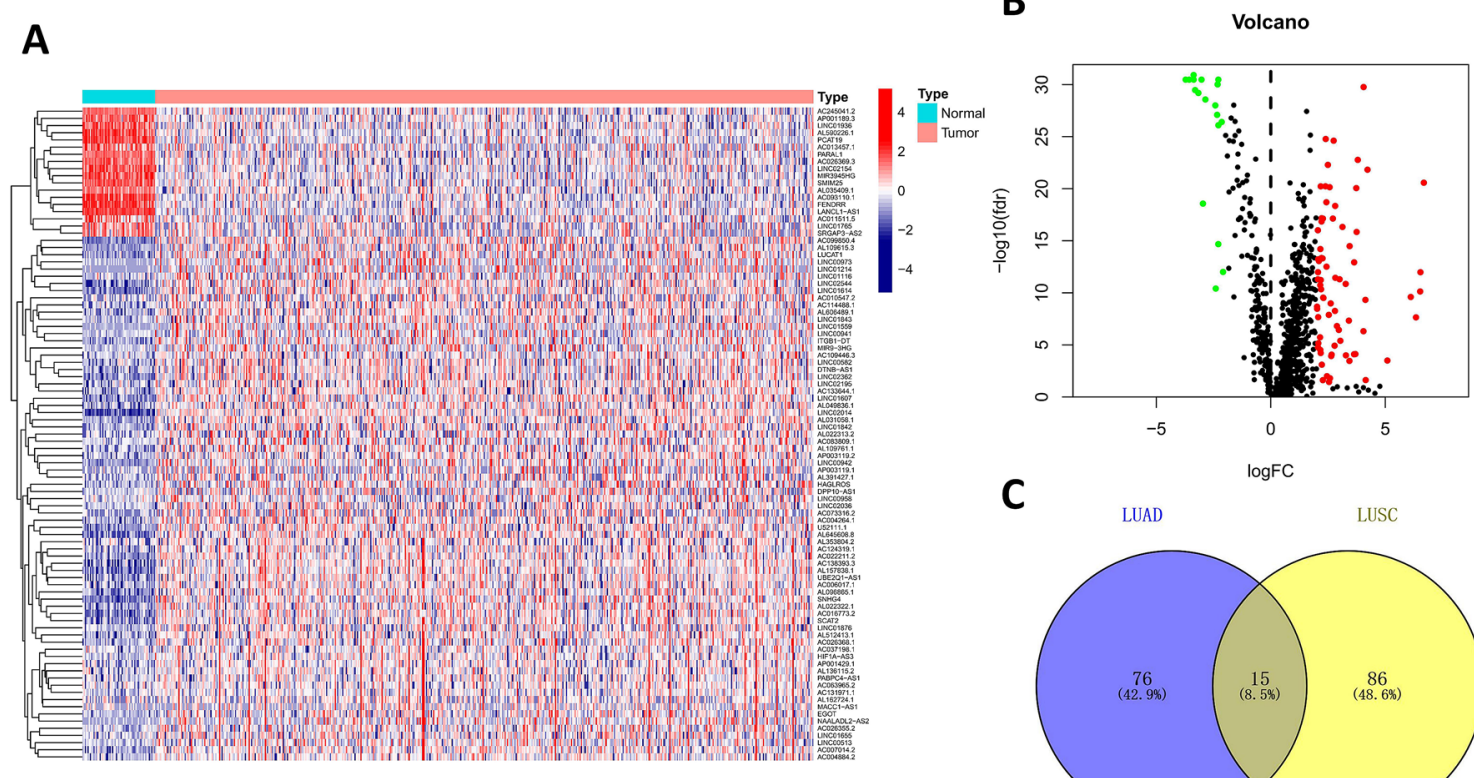

C

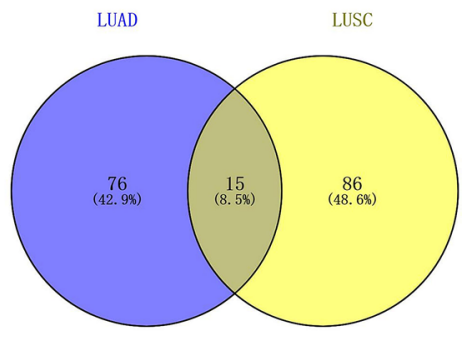

Figure 1. DEirlncRNAs between LUAD samples and normal controls. (A) The heat map of DEirlncRNAs. (B) The volcano plot of DEirlncRNAs. (C) The intersection of DEirlncRNAs between LUAD and LUSC. DEirlncRNAs, differentially expressed immune-related lncRNAs; LUAD, lung adenocarcinoma; LUSC, lung squamous cell carcinoma.

\begin{tabular}{|l|l|l|l|l|l|}
\hline IncRNA pairs & Coef. & HR & HR.95L & HR.95H & p value \\
\hline LINC00958|HIF1A-AS3 & -0.559056064 & 0.571748503 & 0.404824948 & 0.807500506 & 0.00150468 \\
\hline ITGB1-DT|FENDRR & 0.372948657 & 1.452009787 & 1.060841449 & 1.9874152 & 0.019871749 \\
\hline AC004264.1|LINC02036 & 0.39121285 & 1.478773237 & 1.076738936 & 2.030919672 & 0.015661584 \\
\hline AC026355.2|AL049836.1 & -0.42262269 & 0.655325847 & 0.480477131 & 0.893803134 & 0.00760824 \\
\hline LINC02195|LINC01116 & -0.513869512 & 0.598176444 & 0.437208026 & 0.81840917 & 0.00131407 \\
\hline LINC02362|LINC00941 & -0.534861917 & 0.585750167 & 0.411859423 & 0.833059143 & 0.002916854 \\
\hline LINC01116|LINC02154 & 0.621835544 & 1.86234332 & 1.229948538 & 2.819892489 & 0.003305756 \\
\hline AL606489.1|AC006017.1 & 0.443770725 & 1.558573103 & 1.084414844 & 2.240056129 & 0.016491365 \\
\hline
\end{tabular}

Table 2. Immune-related lncRNA pairs used for construction of prognostic model.

Table S2). Besides, we also analyzed the irlncRNAs in lung squamous cell carcinoma (LUSC) and identified 101 DEirlncRNAs between LUSC and normal controls including 62 upregulated and 39 downregulated DEirlncRNAs (Supplementary Figure S1 and Supplementary Table S3 and S4). In addition, the intersection of DEirlncRNAs between LUAD and LUSC was analyzed using the Venny 2.1.0 online database (https://bioinfogp.cnb. csic.es/tools/venny/index.html), and there were only 15 overlapping DEirlncRNAs between LUAD and LUSC (Fig. 1C and Supplementary Table S5). These results indicated that the DEirlncRNAs of LUAD identified in this study had high specificity in LUAD.

Prognostic prediction model of LUAD based on survival-related DEirlncRNA pairs. Totally, 3440 valid DEirlncRNA pairs were identified by the method of cyclically single pairing along with a 0 -or- 1 matrix. Then 619 survival-related DEirlncRNA pairs were determined by univariate Cox regression analysis (Supplementary Table S3). LASSO regression analysis was conducted to screen the optimal survival-related DEirlncRNA pairs, which were used as candidates to construct the prognosis predictive model by multivariate Cox regression analysis. Finally, a total of 8 DEirlncRNA pairs were selected to construct the prediction model of LUAD (Table 2 and Supplementary Table S4).

To validate the accuracy of the model, the 1-, 3-, and 5-year ROC curves were plotted, and they revealed that this model was efficient in predicting the prognosis of LUAD patients since AUC values were all over 0.70 (Fig. 2A). When compared with other clinicopathological characteristics, this risk model possessed the greatest prognostic power with the maximum AUC value of 0.778 in 1-year ROC curve (Fig. 2B). According to the cutoff risk score identified by Youden Index, LUAD patients were divided into high-risk and low-risk subgroups (Fig. 2C). The K-M analysis indicated that this prediction model could efficiently distinguish good or poor 
A

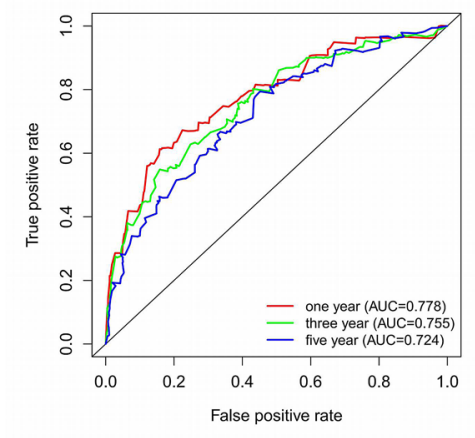

D

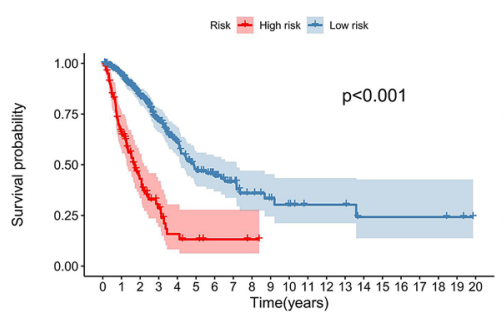

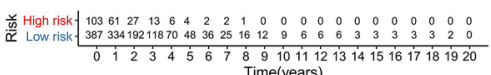

B

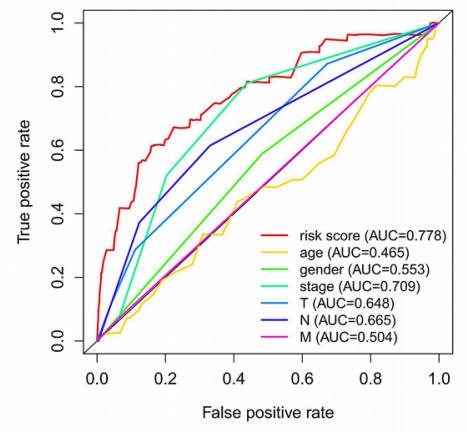

$\mathbf{E}$

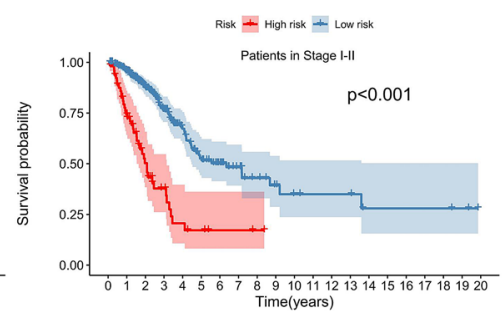

C

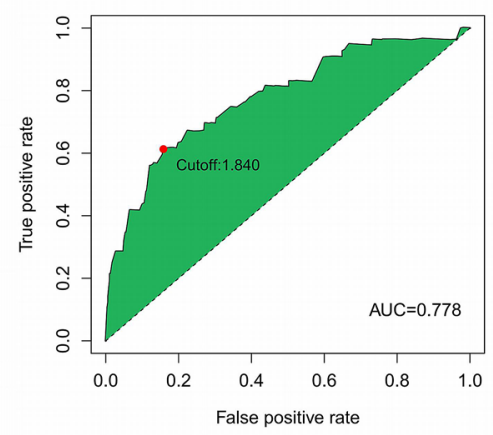

$\mathbf{F}$

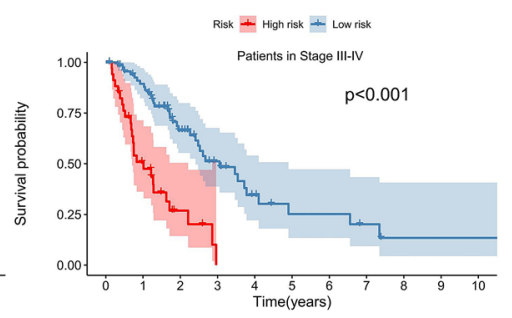

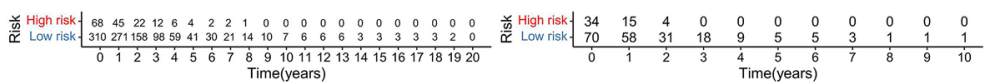

G

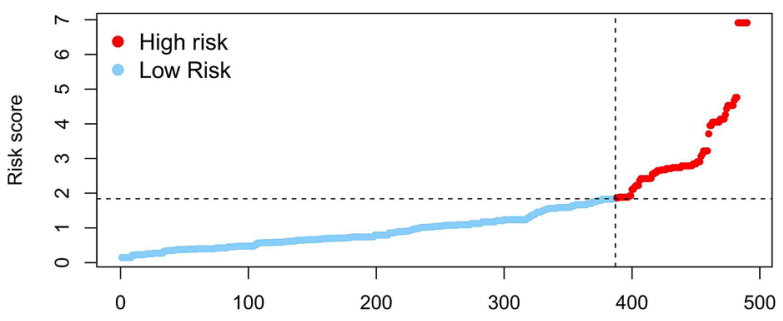

H

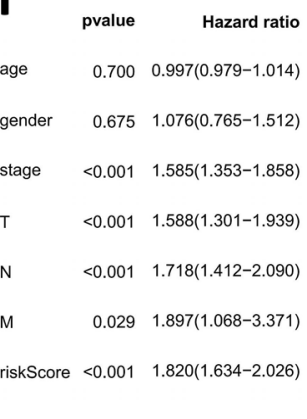

Patients (increasing risk socre)

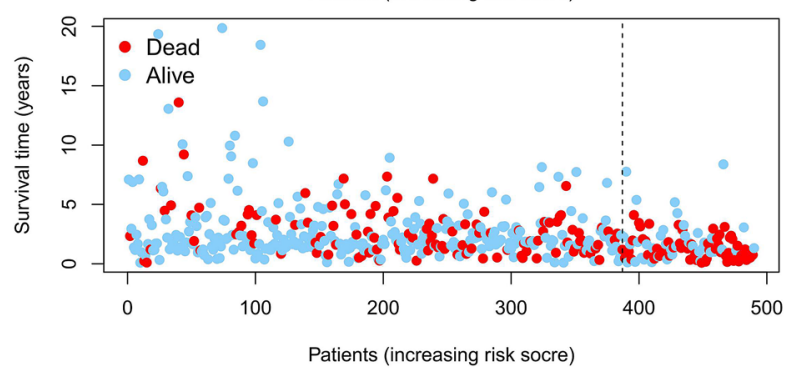

$\begin{array}{lrr} & \text { pvalue } & \text { Hazard ratio } \\ \text { age } & 0.727 & 1.003(0.985-1.022) \\ \text { gender } & 0.229 & 0.803(0.562-1.148) \\ \text { stage } & 0.977 & 1.007(0.643-1.576) \\ \mathrm{T} & 0.061 & 1.262(0.989-1.611) \\ \mathrm{N} & 0.083 & 1.403(0.957-2.058) \\ \mathrm{M} & 0.227 & 2.026(0.644-6.369) \\ \text { riskScore } & <0.001 & 1.755(1.561-1.974)\end{array}$
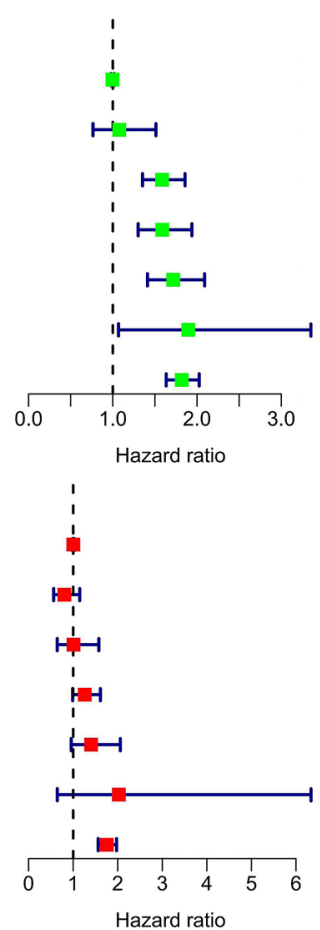

Figure 2. The prognostic prediction model based on survival-related DEirlncRNA pairs in LUAD. (A) The 1-, 3-, and 5-year ROC curves of the risk model with AUC values. (B) The 1-year ROC curve of the risk model and other clinicopathological characteristics. (C) The cut-off risk score identified by Youden Index. (D) The KaplanMeier survival analysis for all LUAD patients. (E) The Kaplan-Meier survival analysis for LUAD patients in Stage I-II. (F) The Kaplan-Meier survival analysis for LUAD patients in Stage III-IV. (G) The risk score curve and the distribution of survival status of LUAD patients. $(\mathrm{H}, \mathbf{I})$ The univariate and multivariate Cox regression analysis to evaluate the independent prognostic value. DEirlncRNAs, differentially expressed immune-related lncRNAs; LUAD, lung adenocarcinoma; ROC, receiver operating characteristic; AUC, area under the curve. 
A

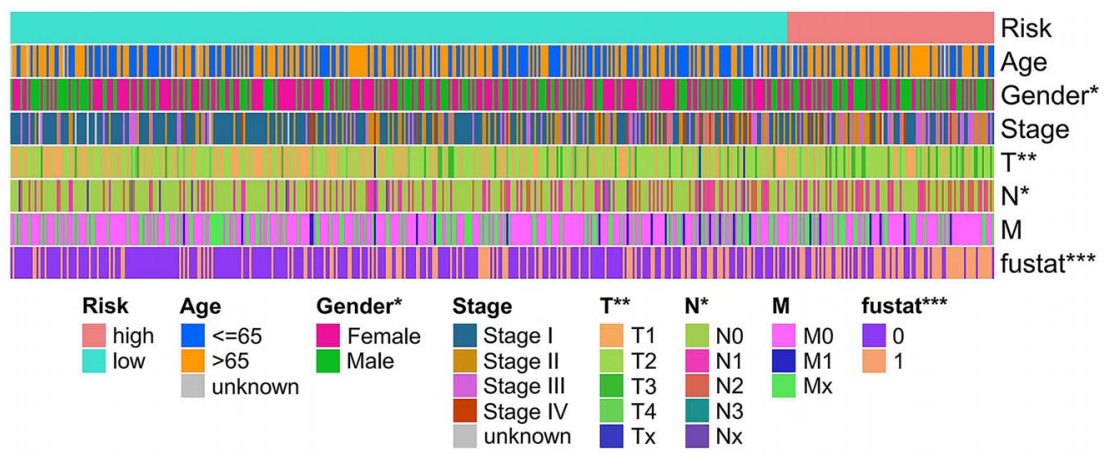

C

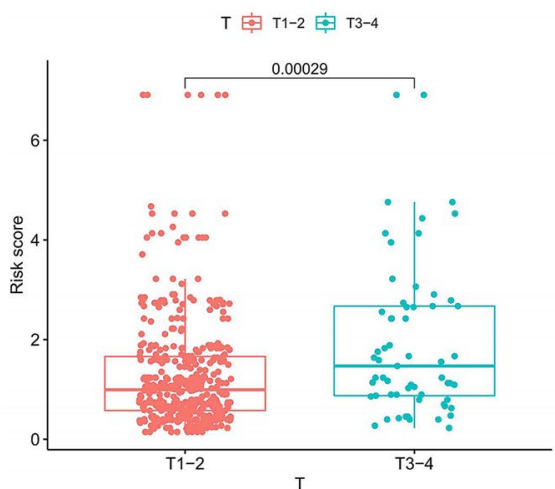

D

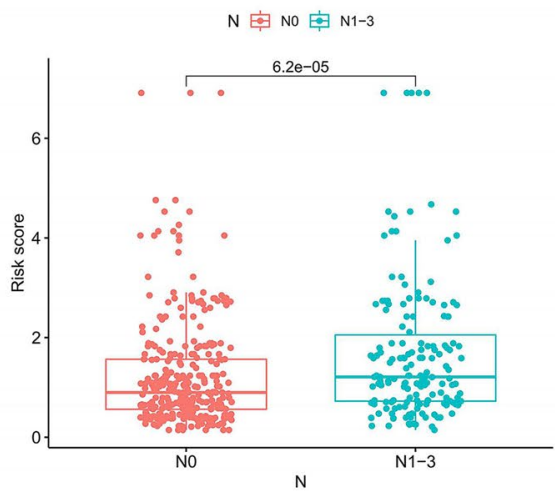

B

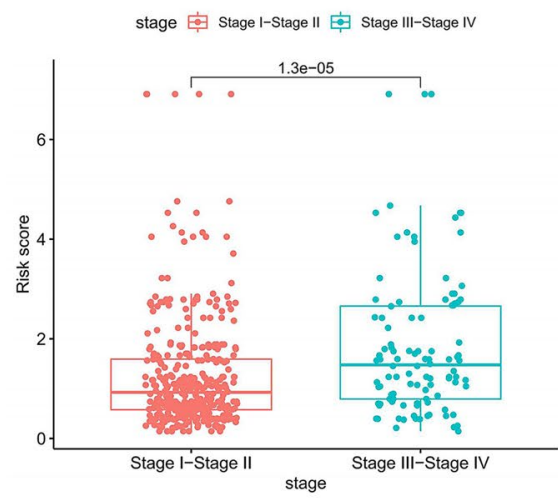

$\mathbf{E}$

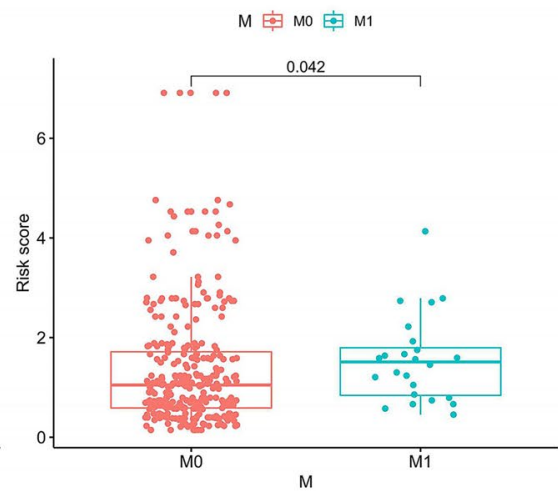

Figure 3. The clinical correlation between risk model and clinicopathological characteristics of LUAD patients. (A) The chi-square test showed that gender $(\mathrm{P}<0.05)$, T stage $(\mathrm{P}<0.01), \mathrm{N}$ stage $(\mathrm{P}<0.05)$, and survival status $(\mathrm{P}<0.001)$ were significantly related to the risk model. (B-E) The Wilcoxon rank-sum test showed that the risk scores of LUAD patients were significantly related to clinical stage (B), status of primary tumor (C), status of lymph node metastasis (D), and status of distant metastasis (E). LUAD, lung adenocarcinoma.

survival of patients with LUAD $(\mathrm{P}<0.001)$ (Fig. 2D). Subgroup analysis was also performed, which showed that this risk model exhibited significant power in distinguishing good or poor survival of LUAD patients not only in Stage I-II subgroup but also in Stage III-IV subgroup (Fig. 2E and 2F). And the risk score curve and the distribution of survival status revealed that high-risk patients had a relatively worse clinical outcome (Fig. 2G). In addition, the univariate Cox regression analysis demonstrated that clinical stage $(\mathrm{p}<0.001)$, T stage $(\mathrm{p}<0.001), \mathrm{N}$ stage $(\mathrm{p}<0.001)$, M stage $(\mathrm{p}=0.029)$ and riskScore $(\mathrm{p}<0.001)$ were significantly associated with poor prognosis, whereas only riskScore $(\mathrm{p}<0.001)$ was statistically different in multivariate Cox regression analysis, implying that only the risk score of the model could serve as an independent prognostic factor for patients with LUAD (Fig. 2H and 2I).

Clinical significance of prognostic prediction model. To estimate the correlation between risk model and clinicopathological characteristics of LUAD patients, we performed chi-square test, which showed that gen$\operatorname{der}(\mathrm{P}<0.05)$, T stage $(\mathrm{P}<0.01)$, N stage $(\mathrm{P}<0.05)$, and survival status $(\mathrm{P}<0.001)$ were significantly related to the risk model (Fig. 3A). Furthermore, according to the Wilcoxon rank-sum test, the risk scores of LUAD patients were significantly related to clinical stage, status of primary tumor, status of lymph node metastasis, and status of distant metastasis (all $\mathrm{P}<0.05)$ (Fig. 3B-3E).

Correlation between risk model and tumor-infiltrating immune cells or immune-related molecules. The correlation between risk score and tumor-infiltrating immune cells was estimated via Spearman correlation analysis, which showed that the risk scores of LUAD patients were more negatively associated with the tumor-infiltrating immune cells, such as B cells, CD8 + T cells, and monocytes. However, some other immune cells, such as M0 macrophages, $\mathrm{CD} 4+\mathrm{T}$ cells, and cancer associated fibroblasts, were positively related to the risk scores of patients with LUAD (Fig. 4A). We also investigated the relationship between the risk model and immune-related molecules and found that the expression levels of CTLA4 gene $(\mathrm{P}<0.01)$ and HAVCR2 gene $(\mathrm{P}<0.05)$ were significantly correlated with the risk model (Fig. 4B-4E). 
A

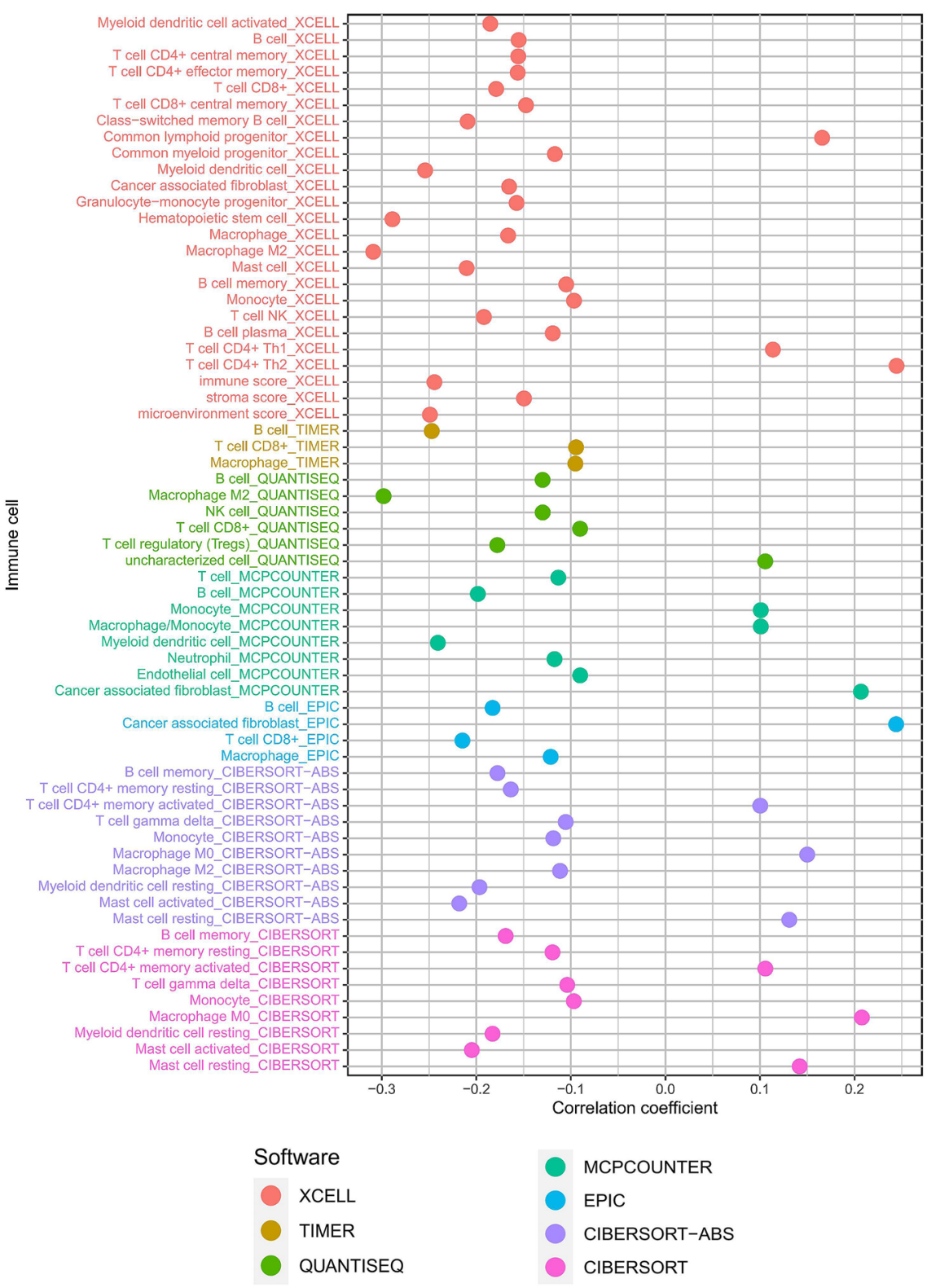

B
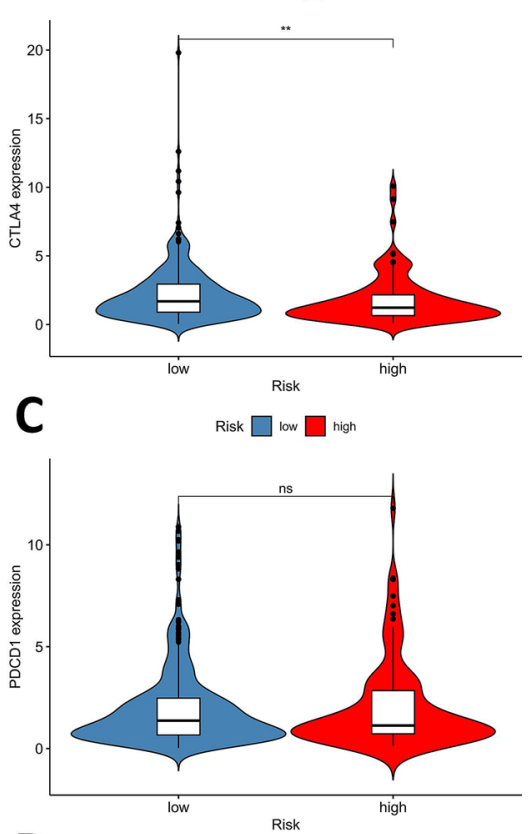

D

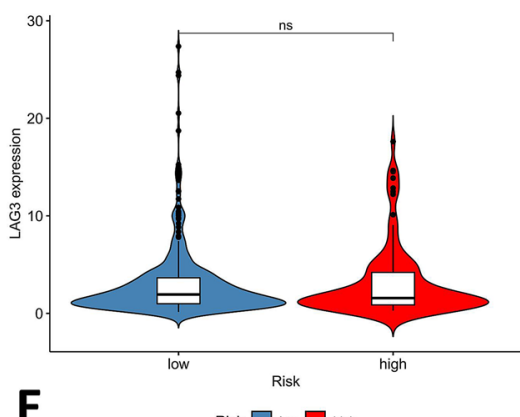

E

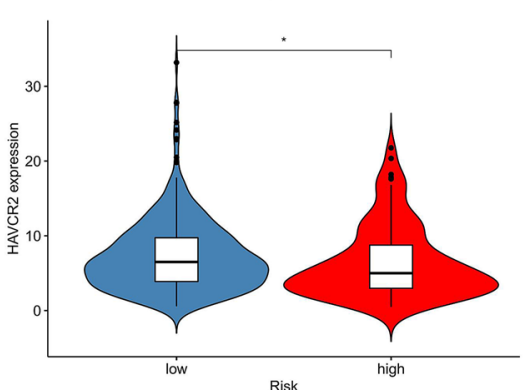

Figure 4. The immune significance of the risk model in LUAD. (A) The correlation between risk score and tumor-infiltrating immune cells, which were analyzed by seven different quantification methods of immune infiltration estimations including TIMER, xCell, quanTIseq, MCP-counter, EPIC, CIBERSORT-ABS, and CIBERSORT. (B-E) The relationship between risk model and the expression levels of immune-related molecules, including CTLA4 (B), PDCD1 (C), LAG3 (D), and HAVCR2 (E). LUAD, lung adenocarcinoma; CTLA4, cytotoxic T-lymphocyte associated protein 4; $P D C D 1$, programmed cell death 1; LAG3, lymphocyte activating 3; HAVCR2, hepatitis A virus cellular receptor 2.

Relationship between risk model and sensitivity of anti-tumor drugs. We used the IPS, IPSCTLA4 blocker, IPS-PD1/PD-L1/PD-L2 blocker, and IPS-CTLA4 and PD1/PD-L1/PD-L2 blocker to assess the potential application values of ICIs for LUAD. The IPS-CTLA4 blocker was significantly higher in the low-risk group $(\mathrm{P}<0.01)$, implying that patients in low-risk subgroup might have a better opportunity for ICIs treatment, especially for the anti-CTLA4 treatment (Fig. 5A-5D). In addition, the sensitivity of anti-tumor drugs (IC50) was calculated and the difference of IC50 between high-risk and low-risk LUAD patients was evaluated. High-risk subgroup was significantly related to higher sensitivity (lower IC50) of anti-tumor drugs including paclitaxel $(\mathrm{P}<0.001)$, docetaxel $(\mathrm{P}<0.001)$, gemcitabine $(\mathrm{P}<0.001)$, vinorelbine $(\mathrm{P}<0.05)$, etoposide $(\mathrm{P}<0.01)$, 
A

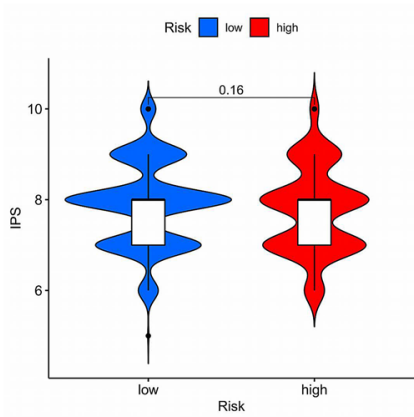

E

Risk 追 low 追 high

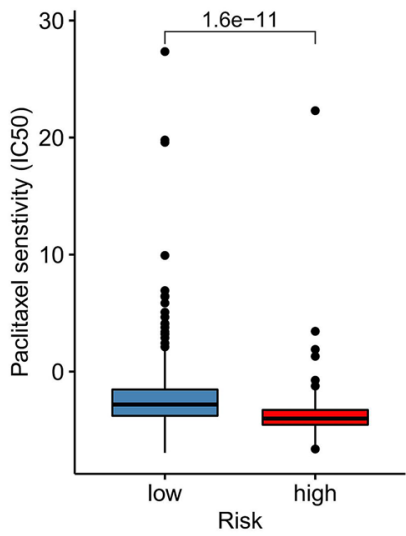

I Risk 追 low 白 high

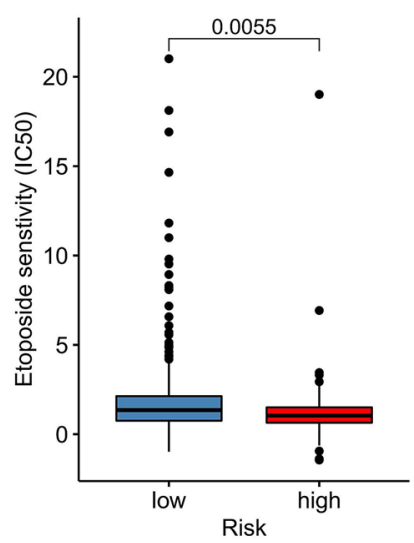

B

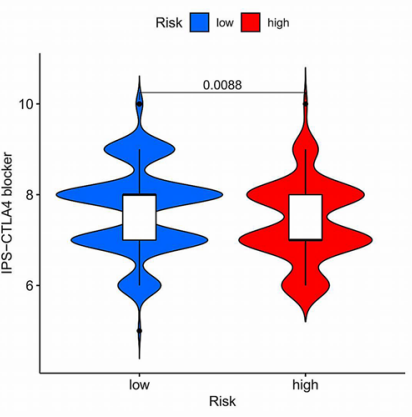

$\mathbf{F}$

Risk 追 low 官 high

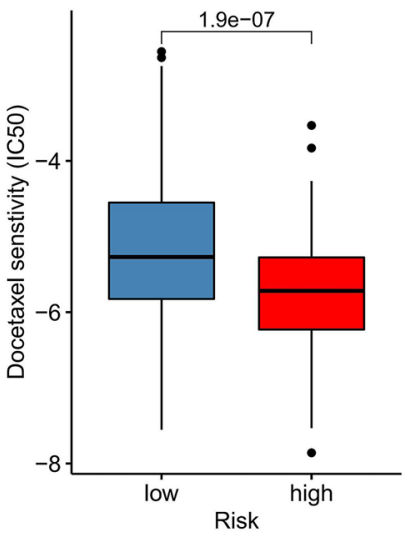

J

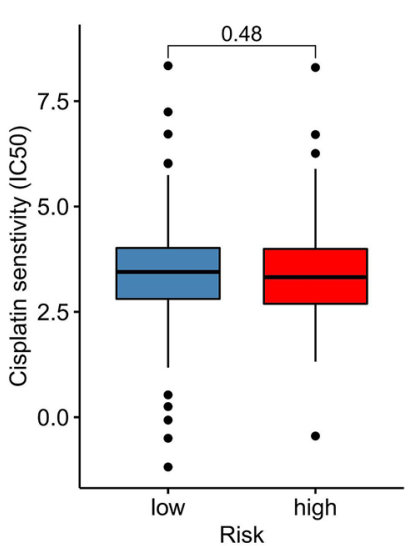

C

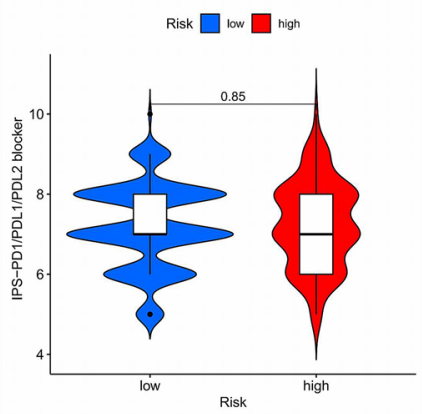

G

Risk 追 low 官 high

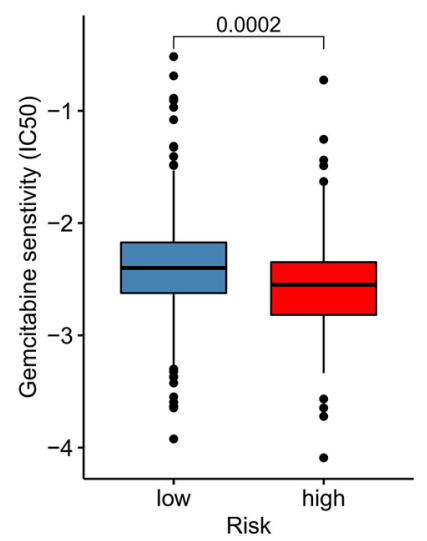

K

Risk 追 low 追 high

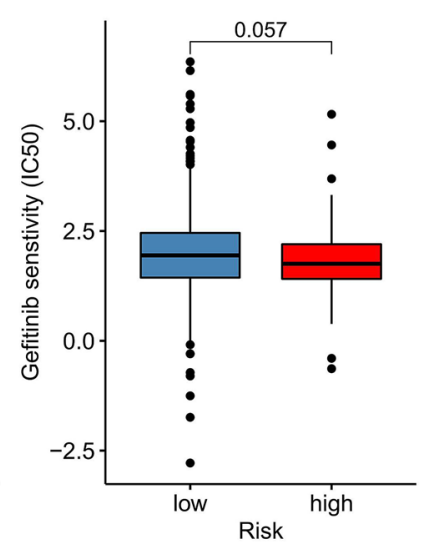

D

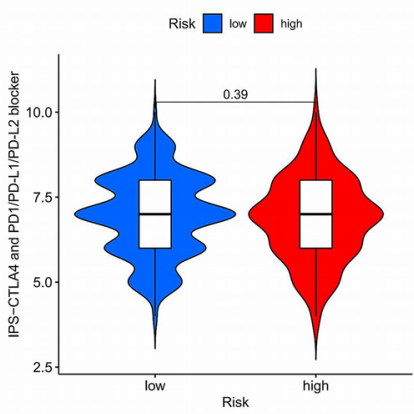

H Risk 追 low 追 high

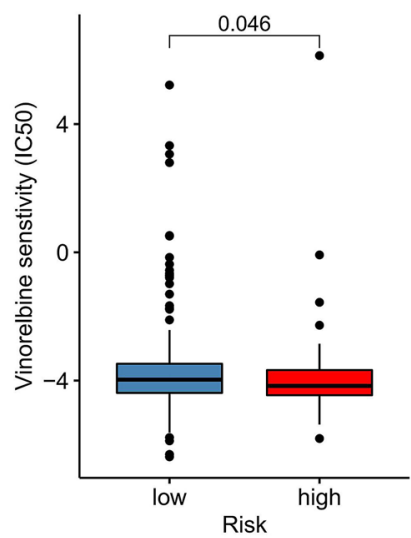

L

Risk 官 low 追 high

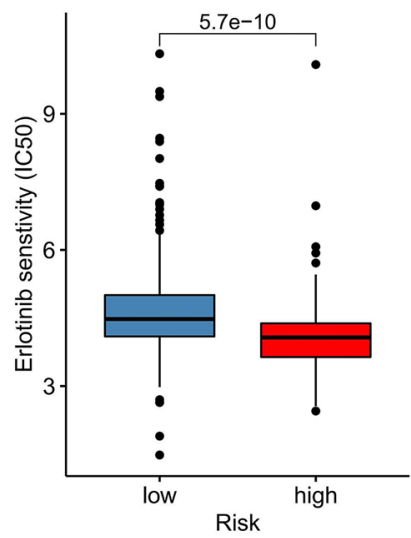

Figure 5. The Relationship between risk model and sensitivity of anti-tumor drugs. (A-D) The association between IPS and the immune-related risk signature of LUAD patients. (E-L) The difference of IC50 of antitumor drugs, including paclitaxel (E), docetaxel (F), gemcitabine (G), vinorelbine (H), etoposide (I), cisplatin (J), gefitinib (K), and erlotinib (L), between the high-risk and low-risk subgroups. IPS, Immunophenoscore; LUAD, lung adenocarcinoma; IC50, the half inhibitory concentration.

and erlotinib $(\mathrm{P}<0.001)$, indicating that this risk model might be used as a biomarker to guide the selection of anti-tumor drugs (Fig. 5E-5L).

\section{Discussion}

LUAD accounts for nearly $40 \%$ of all lung cancer cases, and the prognosis of LUAD patients remains unsatisfactory ${ }^{2,5}$. Therefore, developing effective biomarkers with high specificity and sensitivity is of significance to improve the survival of LUAD patients, especially in the era of immunotherapy. Nowadays, with the development of the technology of high-throughput sequencing, many studies have indicated the prognostic value 
of genome-wide biomarkers in malignant tumors, such as signatures of messenger RNAs (mRNAs), microRNAs (miRNAs), and $\operatorname{lncRNAs} s^{28-30}$. In particular, immune-related signatures have been proved to have effectively predictive values in the treatment and prognosis of cancers including LUAD ${ }^{16-20}$. However, most of these signatures depend on the exact expression levels of transcripts, which weakens their clinical application values due to the heterogeneity of gene expression data.

In this study, we innovatively conducted a new method of cyclically single pairing along with a 0 -or- 1 matrix to construct a novel prognostic signature of irlncRNA pairs in LUAD. This novel signature does not require quantitative expression levels of lncRNAs, but only needs to detect the higher or lower expression level of the two lncRNAs in each lncRNA pair, which improves its clinical practicability. Totally, 8 DEirlncRNA pairs were selected to construct the prediction model of LUAD, which was proved to be efficient to predict the survival of LUAD patients. Among these DEirlncRNAs included in the model, some have been revealed to be related to the development of cancers, such as LINC00958, FOXF1 adjacent non-coding developmental regulatory RNA (FENDRR), LINC01116, and LINC00941.

LncRNA LINC00958 was initially identified as an oncogene in bladder cancer ${ }^{31}$, and subsequent studies revealed the overexpression of LINC00958 in many other malignant tumors, such as hepatocellular carcinoma, pancreatic cancer, gastric cancer, glioma, and cervical cancer ${ }^{32-36}$. In NSCLC, Luo et al. demonstrated that LINC00958 was highly expressed in both LUAD and LUSC cell lines and it could facilitate the proliferation and migration of NSCLC cells, which was mediated by JNK/c-JUN signaling pathway ${ }^{37}$.

LncRNA FENDRR, as a potential tumor suppressor, has been revealed to be downregulated in different cancers, such as gastric cancer, breast cancer, hepatocellular carcinoma as well as NSCLC ${ }^{38-41}$. Zhang et al. demonstrated that FENDRR was downregulated in both NSCLC cells and tissues and was negatively related to the prognosis of NSCLC patients. Up-expression of FENDRR could inhibit the aggressiveness phenotypes of NSCLC cells, such as proliferation, migration and invasion, via directly binding to miR-761 and regulating the expression of tissue inhibitor of metalloproteinases $2(\text { TIMP2 })^{41}$. Besides, Munteanu et al. indicated that FENDRR might also regulate the immune response in macrophages. In detail, the overexpression of FENDRR could enhance interferon $\gamma$ (IFN $\gamma$ ) induced M1 macrophage polarization by modulating signal transducer and activator of transcription 1 (STAT1) activation pathway ${ }^{42}$.

LncRNA LINC01116 was found to be dysregulated in various human cancers, such as glioma, prostate cancer, breast cancer, and osteosarcoma ${ }^{43-46}$. Recent studies also suggested that LINC01116 played an oncogenic role in lung cancer. For instance, Zeng et al. demonstrated the upregulation of LINC01116 in LUAD tissues and cell lines, and short interfering RNAs (siRNAs) induced LINC01116 knockdown could inhibit the cell proliferation, migration, and epithelial-mesenchymal transition (EMT) of LUAD cells ${ }^{47}$. And Wang et al. found that LINC0116 overexpression contributed to cisplatin resistance in $\mathrm{LUAD}^{48}$. Besides, LINC01116 also played a significant role in gefitinib resistance of NSCLC via regulating the expression of interferon-induced protein 4 (IFI4) ${ }^{49}$.

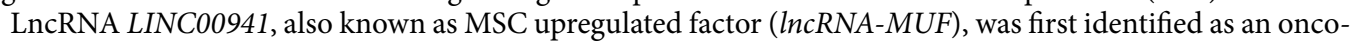
gene in gastric cancer by Luo et al. They found that LINC00941 was overexpression in the tissues of gastric cancer compared with adjacent normal tissues and its aberrant expression was related to invasion depth, TNM stage, and lymphatic metastasis ${ }^{50}$. Consistently, Liu et al. indicated that silence of LINC00941 could inhibit the proliferation, migration, and invasion of gastric cancer cells ${ }^{51}$. Meanwhile, Wang et al. found that LINC00941 could act as a competing endogenous RNA (ceRNA) by sponging miR-335-5p to regulate ROCK1-mediated LIMK1/ Cofilin-1 signaling, which contributed to the proliferation, migration, invasion, and EMT of pancreatic cancer cells ${ }^{52}$. And in LUAD, LINC0094 could regulate focal adhesion and PI3K-AKT signaling pathway, and its elevated expression level was related to decreased survival of LUAD patients ${ }^{53}$. However, in this study, the potential regulatory mechanisms of these lncRNAs were not clearly elucidated. LncRNAs play significant roles in maintaining physiological functions by regulating gene expression at both the transcriptional and post-transcriptional levels. Nowadays, Salmena et al. put forward a competing endogenous RNA (ceRNA) hypothesis, in which lncRNAs, mRNAs, and other RNAs were able to compete with each other as ceRNAs to bind to miRNAs through sharing one or more miRNA response elements (MREs) ${ }^{54}$. LncRNAs can serve as endogenous molecular sponges for miRNAs to indirectly regulate the expression of mRNAs. For instance, Zhao et al. have indicated that lncRNA HOMEOBOX A11 antisense RNA (HOXA11-AS), acting as a ceRNA by sponging miR-454-3p, could regulate the expression of signal transducer and activator of transcription 3 (STAT3), and the HOXA11-AS/miR-454-3p/ Stat 3 axis was associated with the cisplatin resistance of human LUAD cells ${ }^{55}$. Uncovering the potential regulatory roles of lncRNAs, including its regulation on immune system and other pathological and physiological processes, may provide new insights into the pathological mechanisms in LUAD.

To evaluate the efficacy and accuracy of this prediction model, we performed 1-, 3-, and 5-year ROC curve analysis and the results showed that this model was efficient in predicting the prognosis of LUAD patients since AUC values were all over 0.70 . And based on the optimal cut-off risk score identified by Youden Index, LUAD patients were divided into high-risk and low-risk subgroups, and K-M analysis revealed that this risk model exhibited great power in distinguishing good or poor survival of LUAD patients. In addition, the risk score of the model was found to be an independent prognostic factor for LUAD patients. Moreover, in order to estimate the clinical significance of the model, we performed chi-square test and Wilcoxon rank-sum test to explore the correlation between risk model and clinicopathological characteristics of LUAD patients, which indicated that the risk model was significantly associated with the clinical stage of patients including $\mathrm{T}$ stage, $\mathrm{N}$ stage, and $\mathrm{M}$ stage. All these results implied that this risk model performed well in predicting the prognosis of LUAD patients and these survival-related irlncRNAs included in the model might be used as novel therapeutic targets for LUAD treatment in the future.

In recent years, the treatment of lung cancer has entered the era of immunotherapy. However, not all patients with lung cancer can benefit from the treatment of immunotherapeutic agents, and the response rate of LUAD patients to immunotherapy remains unsatisfactory ${ }^{5}$. Tumor infiltrating immune cells in TME participate in 
various biological processes of malignant tumors, and the interaction between infiltrating immune cells and cancer cells can influence the malignant phenotypes of cancers ${ }^{56,57}$. In this study, we comprehensively estimated the tumor-infiltrating immune cells of LUAD samples by using seven acceptable methods including xCell, TIMER, quanTIseq, MCP-counter, EPIC, CIBERSORT-ABS, and CIBERSORT ${ }^{25-27}$, and then analyzed the relationship between tumor-infiltrating immune cells and the risk model. The correlation analysis indicated that the high-risk subgroup was more negatively related to tumor-infiltrating immune cells, such as CD8 + T cells and monocytes. CD8 + T cells are key effectors in anti-tumor immunity, and the frequency of CD8 + T cells is positively associated with the survival of patients with lung cancer, melanoma, and breast cancer ${ }^{58,59}$. In addition, the infiltration of CD8 + T cells in TME is related to improved responses of cancer patients treated with ICIs. For instance, Wong et al. demonstrated that melanoma patients with high CD8 + T cell count experienced prolonged survival when treated with anti-PD-1 therapy ${ }^{60}$. In addition, monocytes are a subtype of innate immune cells, which also play significant roles in anti-tumor immunity by various mechanisms, such as phagocytosis, apoptosis, and cell contact-mediated antibody-dependent cellular cytotoxicity (ADCC) ${ }^{61,62}$. The role of CD8 + T cells and monocytes in anti-tumor immunity was consistent with our results of correlation analysis, which indicated that LUAD patients in low-risk subgroup have more CD8 $+\mathrm{T}$ cells and monocytes infiltration. Besides, we also found that the risk scores of LUAD patients were significantly related to the expression levels of CTLA4 gene and HAVCR2 gene, which have been proved to be potential biomarkers associated with the treatment of ICIs ${ }^{63,64}$.

To further evaluate the clinical application value of the risk model in the treatment of LUAD, we calculated the IC50 of several common anti-tumor drugs and compared the differences of drug sensitivity between patients with high-risk and low-risk subgroups. We found that high-risk LUAD patients have higher sensitivity (lower IC50) of anti-tumor drugs including paclitaxel, docetaxel, gemcitabine, vinorelbine, etoposide, and erlotinib. This relationship between risk model and drug sensitivity might be used to guide the selection and administration of anti-tumor drugs in clinical practice, which needs to be further investigated in the future.

However, there are some shortcomings in this study. First, the RNA-seq data of LUAD cohort was only downloaded from TCGA database. Although we performed various methods to validate the accuracy and efficiency of our prognostic prediction model, additional external cohorts are needed to confirm it in the future. In addition, the expression of these irlncRNAs in lung tissues of LUAD patients need to be further investigated, such as RNA fluorescence in situ hybridization (FISH) and histological staining. And the potential regulatory mechanisms of lncRNAs and the relationship between lncRNAs and immune system need to be further studied. Our team will perform biological experiments and clinical studies to explore the downstream signaling pathways of these lncRNAs in the future. Second, the tumor-infiltrating immune cells of LUAD samples were estimated by different quantification methods based on the RNA-seq data, which need to be experimentally validated. Finally, the clinical application value of our risk model, such as its relationship with anti-tumor drug sensitivity, has not been clinically verified. Further studies with larger sample sizes of LUAD patients are required to confirm our results in the future.

In conclusion, we innovatively constructed a novel signature of DEirlncRNA pairs in LUAD, which did not depend on specific expression levels of lncRNAs. This signature performed well in predicting the prognosis of LUAD patients and might be used to guide the administration of patients with LUAD in clinical practice. Future studies, preferably with a large sample size, are needed to verify our findings.

Received: 2 April 2021; Accepted: 6 August 2021

Published online: 18 August 2021

\section{References}

1. Siegel, R. L., Miller, K. D., Fuchs, H. E. \& Jemal, A. Cancer statistics, 2021. CA: J. Clin. 71, 7-33. https://doi.org/10.3322/caac. 21654 (2021).

2. Bade, B. C., Dela Cruz, C. S. \& Cancer, L. Epidemiology, etiology, and prevention. Clin. Chest Med. 41(1-24), 2020. https://doi. org/10.1016/j.ccm.2019.10.001 (2020).

3. Martinez, P., Peters, S., Stammers, T. \& Soria, J. C. Immunotherapy for the first-line treatment of patients with metastatic non-small cell lung cancer. Clin. Cancer Res. 25, 2691-2698. https://doi.org/10.1158/1078-0432.CCR-18-3904 (2019).

4. Lin, J. J. et al. Five-year survival in EGFR -mutant metastatic lung adenocarcinoma treated with EGFR-TKIs. J. Thorac. Oncol. 11, 556-565. https://doi.org/10.1016/j.jtho.2015.12.103 (2016).

5. Heist, R. S. \& Engelman, J. A. SnapShot: non-small cell lung cancer. Cancer Cell 21, 448. https://doi.org/10.1016/j.ccr.2012.03.007 (2012).

6. Hanahan, D. \& Coussens, L. M. Accessories to the crime: functions of cells recruited to the tumor microenvironment. Cancer Cell 21, 309-322. https://doi.org/10.1016/j.ccr.2012.02.022 (2012).

7. Quail, D. F. \& Joyce, J. A. Microenvironmental regulation of tumor progression and metastasis. Nat. Med. 19, 1423-1437. https:// doi.org/10.1038/nm.3394 (2013).

8. Dunn, G. P., Bruce, A. T., Ikeda, H., Old, L. J. \& Schreiber, R. D. Cancer immunoediting: from immunosurveillance to tumor escape. Nat Immunol 3, 991-998. https://doi.org/10.1038/ni1102-991 (2002).

9. Kolahian, S. et al. The emerging role of myeloid-derived suppressor cells in lung diseases. Eur. Respir. J. 47, 967-977. https://doi. org/10.1183/13993003.01572-2015 (2016).

10. Zhang, D., Chen, Z., Wang, D. C. \& Wang, X. Regulatory T cells and potential inmmunotherapeutic targets in lung cancer. Cancer Metastasis Rev. 34, 277-290. https://doi.org/10.1007/s10555-015-9566-0 (2015).

11. Mercer, T. R., Dinger, M. E. \& Mattick, J. S. Long non-coding RNAs: insights into functions. Nat. Rev. Genet. 10, 155-159. https:// doi.org/10.1038/nrg2521 (2009).

12. Shi, X., Sun, M., Liu, H., Yao, Y. \& Song, Y. Long non-coding RNAs: a new frontier in the study of human diseases. Cancer Lett 339, 159-166. https://doi.org/10.1016/j.canlet.2013.06.013 (2013).

13. Chen, Y. G., Satpathy, A. T. \& Chang, H. Y. Gene regulation in the immune system by long noncoding RNAs. Nat Immunol 18, 962-972. https://doi.org/10.1038/ni.3771 (2017). 
14. Hu, Q. et al. Oncogenic lncRNA downregulates cancer cell antigen presentation and intrinsic tumor suppression. Nat Immunol 20, 835-851. https://doi.org/10.1038/s41590-019-0400-7 (2019).

15. Li, Y. et al. Pan-cancer characterization of immune-related lncRNAs identifies potential oncogenic biomarkers. Nat. Commun. 11, 1000. https://doi.org/10.1038/s41467-020-14802-2 (2020).

16. Lin, Y., Pan, X., Chen, Z., Lin, S. \& Chen, S. Identification of an immune-related nine-lncRNA signature predictive of overall survival in colon cancer. Front. Genet. 11, 318. https://doi.org/10.3389/fgene.2020.00318 (2020).

17. Wei, C. et al. Bioinformatics profiling utilized a nine immune-related long noncoding RNA signature as a prognostic target for pancreatic cancer. J. Cell. Biochem. 120, 14916-14927. https://doi.org/10.1002/jcb.28754 (2019).

18. Miao, H. et al. Identification of an immune-related six-long noncoding RNA signature as a novel prognosis biomarker for adenocarcinoma of lung. Biosci Rep. https://doi.org/10.1042/BSR20202444 (2021).

19. Shen, Y., Peng, X. \& Shen, C. Identification and validation of immune-related lncRNA prognostic signature for breast cancer. Genomics 112, 2640-2646. https://doi.org/10.1016/j.ygeno.2020.02.015 (2020).

20. Li, J. P. et al. A seven immune-related lncRNAs model to increase the predicted value of lung adenocarcinoma. Front. Oncol. 10, 560779. https://doi.org/10.3389/fonc.2020.560779 (2020).

21. Ritchie, M. E. et al. limma powers differential expression analyses for RNA-sequencing and microarray studies. Nucleic Acids Res. 43, e47. https://doi.org/10.1093/nar/gkv007 (2015).

22. Glickman, M. E., Rao, S. R. \& Schultz, M. R. False discovery rate control is a recommended alternative to Bonferroni-type adjustments in health studies. J Clin Epidemiol 67, 850-857. https://doi.org/10.1016/j.jclinepi.2014.03.012 (2014).

23. Sauerbrei, W., Royston, P. \& Binder, H. Selection of important variables and determination of functional form for continuous predictors in multivariable model building. Stat. Med. 26, 5512-5528. https://doi.org/10.1002/sim.3148 (2007).

24. Youden, W. J. Index for rating diagnostic tests. Cancer 3, 32-35. https://doi.org/10.1002/1097-0142(1950)3:1\%3c32::aid-cncr2 820030106\%3e3.0.co;2-3 (1950).

25. Li, T. et al. TIMER2.0 for analysis of tumor-infiltrating immune cells. Nucleic Acids Res. 48, W509-W514. https://doi.org/10.1093/ nar/gkaa407 (2020).

26. Li, T. et al. TIMER: A web server for comprehensive analysis of tumor-infiltrating immune cells. Can. Res. 77, e108-e110. https:// doi.org/10.1158/0008-5472.CAN-17-0307 (2017).

27. Sturm, G. et al. Comprehensive evaluation of transcriptome-based cell-type quantification methods for immuno-oncology. Bioinformatics 35, i436-i445. https://doi.org/10.1093/bioinformatics/btz363 (2019).

28. Rokavec, M., Horst, D. \& Hermeking, H. Cellular model of colon cancer progression reveals signatures of mRNAs, miRNA, lncRNAs, and epigenetic modifications associated with metastasis. Can. Res. 77, 1854-1867. https://doi.org/10.1158/0008-5472. CAN-16-3236 (2017).

29. Nie, W. et al. LncRNA-UCA1 exerts oncogenic functions in non-small cell lung cancer by targeting miR-193a-3p. Cancer Lett 371, 99-106. https://doi.org/10.1016/j.canlet.2015.11.024 (2016).

30. Zhao, X., Sun, S., Zeng, X. \& Cui, L. Expression profiles analysis identifies a novel three-mRNA signature to predict overall survival in oral squamous cell carcinoma. Am. J. Cancer Res. 8, 450-461 (2018).

31. Seitz, A. K. et al. Profiling of long non-coding RNAs identifies LINC00958 and LINC01296 as candidate oncogenes in bladder cancer. Sci Rep 7, 395. https://doi.org/10.1038/s41598-017-00327-0 (2017).

32. Zuo, X. et al. M6A-mediated upregulation of LINC00958 increases lipogenesis and acts as a nanotherapeutic target in hepatocellular carcinoma. J. Hematol. Oncol. 13, 5. https://doi.org/10.1186/s13045-019-0839-x (2020).

33. Chen, S. et al. Silencing of long noncoding RNA LINC00958 prevents tumor initiation of pancreatic cancer by acting as a sponge of microRNA-330-5p to down-regulate PAX8. Cancer Lett. 446, 49-61. https://doi.org/10.1016/j.canlet.2018.12.017 (2019).

34. Wang, W. et al. Elevated long non-coding RNA LINC00958 was associated with metastasis and unfavorable prognosis in gastric cancer. Eur. Rev. Med. Pharmacol. Sci. 23, 598-603. https://doi.org/10.26355/eurrev_201901_16872 (2019).

35. Guo, E. et al. Long noncoding RNA LINC00958 accelerates gliomagenesis through regulating miR-203/CDK2. DNA Cell Biol. 37, 465-472. https://doi.org/10.1089/dna.2018.4163 (2018).

36. Wang, L. et al. LINC00958 facilitates cervical cancer cell proliferation and metastasis by sponging miR-625-5p to upregulate LRRC8E expression. J. Cell. Biochem. 121, 2500-2509. https://doi.org/10.1002/jcb.29472 (2020).

37. Luo, Z., Han, Z., Shou, F., Li, Y. \& Chen, Y. LINC00958 accelerates cell proliferation and migration in non-small cell lung cancer through JNK/c-JUN signaling. Hum. Gene Ther. Methods 30, 226-234. https://doi.org/10.1089/hgtb.2019.115 (2019).

38. Xu, T. P. et al. Decreased expression of the long non-coding RNA FENDRR is associated with poor prognosis in gastric cancer and FENDRR regulates gastric cancer cell metastasis by affecting fibronectin1 expression. J. Hematol. Oncol. 7, 63. https://doi.org/10. 1186/s13045-014-0063-7 (2014).

39. Li, Y. et al. Long non-coding RNA FENDRR inhibits cell proliferation and is associated with good prognosis in breast cancer. Onco. Targets. Ther. 11, 1403-1412. https://doi.org/10.2147/OTT.S149511 (2018).

40. Wang, B. et al. Long non-coding RNA FENDRR inhibits proliferation and invasion of hepatocellular carcinoma by down-regulating glypican-3 expression. Biochem. Biophys. Res. Commun. 509, 143-147. https://doi.org/10.1016/j.bbrc.2018.12.091 (2019).

41. Zhang, G., Wang, Q., Zhang, X., Ding, Z. \& Liu, R. LncRNA FENDRR suppresses the progression of NSCLC via regulating miR761/TIMP2 axis. Biomed. Pharmacother. 118, 109309. https://doi.org/10.1016/j.biopha.2019.109309 (2019).

42. Munteanu, M. C. et al. Long non-coding RNA FENDRR regulates IFNgamma-induced M1 phenotype in macrophages. Sci Rep 10, 13672. https://doi.org/10.1038/s41598-020-70633-7 (2020).

43. Ye, J. et al. A novel IncRNA-LINC01116 regulates tumorigenesis of glioma by targeting VEGFA. Int. J. Cancer 146, 248-261. https:// doi.org/10.1002/ijc.32483 (2020).

44. Hu, H. B., Chen, Q. \& Ding, S. Q. LncRNA LINC01116 competes with miR-145 for the regulation of ESR1 expression in breast cancer. Eur. Rev. Med. Pharmacol. Sci. 22, 1987-1993. https://doi.org/10.26355/eurrev_201804_14726 (2018).

45. Beaver, L. M. et al. Long noncoding RNAs and sulforaphane: a target for chemoprevention and suppression of prostate cancer. J. Nutr. Biochem. 42, 72-83. https://doi.org/10.1016/j.jnutbio.2017.01.001 (2017).

46. Zhang, Z. F., Xu, H. H., Hu, W. H., Hu, T. Y. \& Wang, X. B. LINC01116 promotes proliferation, invasion and migration of osteosarcoma cells by silencing p53 and EZH2. Eur. Rev. Med. Pharmacol. Sci. 23, 6813-6823. https://doi.org/10.26355/eurrev_201908_ 18720 (2019).

47. Zeng, L. et al. Long non-coding RNA LINC01116 is overexpressed in lung adenocarcinoma and promotes tumor proliferation and metastasis. Am. J. Transl. Res. 12, 4302-4313 (2020).

48. Wang, J. et al. LncRNA LINC01116 contributes to cisplatin resistance in lung adenocarcinoma. Onco. Targets. Ther. 13, 9333-9348. https://doi.org/10.2147/OTT.S244879 (2020).

49. Wang, H. et al. Long Noncoding RNA LINC01116 contributes to gefitinib resistance in non-small cell lung cancer through regulating IFI44. Mol. Ther. Nucleic Acids 19, 218-227. https://doi.org/10.1016/j.omtn.2019.10.039 (2020).

50. Luo, C. et al. Regulatory network analysis of high expressed long non-coding RNA LINC00941 in gastric cancer. Gene 662, 103-109. https://doi.org/10.1016/j.gene.2018.04.023 (2018).

51. Liu, H. et al. Long non-coding RNA LINC00941 as a potential biomarker promotes the proliferation and metastasis of gastric cancer. Front. Genet. 10, 5. https://doi.org/10.3389/fgene.2019.00005 (2019). 
52. Wang, J., He, Z., Xu, J., Chen, P. \& Jiang, J. Long noncoding RNA LINC00941 promotes pancreatic cancer progression by competitively binding miR-335-5p to regulate ROCK1-mediated LIMK1/Cofilin-1 signaling. Cell Death Dis. 12, 36. https://doi.org/ 10.1038/s41419-020-03316-w (2021).

53. Wang, L. et al. Systematic identification of lincRNA-based prognostic biomarkers by integrating lincRNA expression and copy number variation in lung adenocarcinoma. Int. J. Cancer 144, 1723-1734. https://doi.org/10.1002/ijc.31865 (2019).

54. Salmena, L., Poliseno, L., Tay, Y., Kats, L. \& Pandolfi, P. P. A ceRNA Hypothesis: The Rosetta Stone of a Hidden RNA Language?. Cell 146, 353-358. https://doi.org/10.1016/j.cell.2011.07.014 (2011).

55. Zhao, X. et al. LncRNA HOXA11-AS drives cisplatin resistance of human LUAD cells via modulating miR-454-3p/Stat3. Cancer Sci. 109, 3068-3079. https://doi.org/10.1111/cas.13764 (2018).

56. Gajewski, T. F., Schreiber, H. \& Fu, Y. X. Innate and adaptive immune cells in the tumor microenvironment. Nat Immunol 14, 1014-1022. https://doi.org/10.1038/ni.2703 (2013).

57. Man, Y. G. et al. Tumor-infiltrating immune cells promoting tumor invasion and metastasis: existing theories. J Cancer 4, 84-95. https://doi.org/10.7150/jca.5482 (2013).

58. Reiser, J. \& Banerjee, A. Effector, memory, and dysfunctional CD8(+) T cell fates in the antitumor immune response. J. Immunol. Res. 2016, 8941260. https://doi.org/10.1155/2016/8941260 (2016).

59. van der Leun, A. M., Thommen, D. S. \& Schumacher, T. N. CD8(+) T cell states in human cancer: insights from single-cell analysis. Nat. Rev. Cancer 20, 218-232. https://doi.org/10.1038/s41568-019-0235-4 (2020).

60. Wong, P. F. et al. Multiplex quantitative analysis of tumor-infiltrating lymphocytes and immunotherapy outcome in metastatic melanoma. Clin. Cancer Res. 25, 2442-2449. https://doi.org/10.1158/1078-0432.CCR-18-2652 (2019).

61. Olingy, C. E., Dinh, H. Q. \& Hedrick, C. C. Monocyte heterogeneity and functions in cancer. J. Leukoc. Biol. 106, 309-322. https:// doi.org/10.1002/JLB.4RI0818-311R (2019).

62. Gouveia-Fernandes, S. Monocytes and macrophages in cancer: Unsuspected roles. Adv. Exp. Med. Biol. 1219, 161-185. https:// doi.org/10.1007/978-3-030-34025-4_9 (2020).

63. Pistillo, M. P. et al. Phenotypic characterization of tumor CTLA-4 expression in melanoma tissues and its possible role in clinical response to Ipilimumab. Clin. Immunol. 215, 108428. https://doi.org/10.1016/j.clim.2020.108428 (2020).

64. Solinas, C., De Silva, P., Bron, D., Willard-Gallo, K. \& Sangiolo, D. Significance of TIM3 expression in cancer: From biology to the clinic. Semin Oncol 46, 372-379. https://doi.org/10.1053/j.seminoncol.2019.08.005 (2019).

\section{Acknowledgements}

This research was supported by the National Natural Science Foundation of China (No. 81572288), the National Key Research and Development Projects of China (Nos. 2016YEE0103400 and 2018YFC1312201), and the Academic Promotion Program of Shandong First Medical University (No. 2019ZL002).

\section{Author contributions}

QZ and JY contributed to conception and design of the study. YL and QW performed data analysis of the study. $\mathrm{YL}, \mathrm{XF}$, and WL contributed to the software analysis. YL prepared the original manuscript. QW, XF, WL, XL and $\mathrm{HZ}$ reviewed and edited the manuscript. All authors approved the submitted version.

\section{Competing interests}

The authors declare no competing interests.

\section{Additional information}

Supplementary Information The online version contains supplementary material available at https://doi.org/ 10.1038/s41598-021-96236-4.

Correspondence and requests for materials should be addressed to Q.Z. or J.Y.

Reprints and permissions information is available at www.nature.com/reprints.

Publisher's note Springer Nature remains neutral with regard to jurisdictional claims in published maps and institutional affiliations.

Open Access This article is licensed under a Creative Commons Attribution 4.0 International License, which permits use, sharing, adaptation, distribution and reproduction in any medium or format, as long as you give appropriate credit to the original author(s) and the source, provide a link to the Creative Commons licence, and indicate if changes were made. The images or other third party material in this article are included in the article's Creative Commons licence, unless indicated otherwise in a credit line to the material. If material is not included in the article's Creative Commons licence and your intended use is not permitted by statutory regulation or exceeds the permitted use, you will need to obtain permission directly from the copyright holder. To view a copy of this licence, visit http://creativecommons.org/licenses/by/4.0/.

(C) The Author(s) 2021 